\title{
Synthesis of Some New Biologically Active Benzothiazole Derivatives Containing Benzimidazole and Imidazoline Moieties
}

\author{
Manish Chaudhary, Deepak Pareek, Pawan K. Pareek, ${ }^{\dagger}$ Ravi Kant, ${ }^{\dagger}$ Krishan G Ojha, ${ }^{\dagger}$ and Arun Pareek ${ }^{*}$ \\ Analytical \& Pharmaceutical Research Laboratory, Department of Chemistry, Government College Ajmer, 305001 India \\ ${ }^{*}$ E-mail: arunpareek7@gmail.com \\ ${ }^{\dagger}$ Department of Pure and Applied Chemistry, M. D. S. University, Ajmer 305009 India \\ ${ }^{\ddagger}$ Hygia Institutes of Pharmaceutical Education and Research, Lucknow, 226020 India \\ Received June 30, 2010, Accepted November 2, 2010
}

\begin{abstract}
Synthesis of $N$-(1H-benzimidazol-2-yl)-6-substituted-1,3-benzothiazol-2-amines and 6-substituted- $N$-(4,5-dihydro$1 H$-imidazol-2-yl)-1,3-benzothiazol-2-amines by the reaction of substituted 2-aminobenzothiazoles with carbon disulphide and methyl iodide followed by the reaction with $o$-phenylene diamine/ethylene diamine are reported. All the synthesized compounds were characterized by elemental analysis, IR spectra and ${ }^{1} \mathrm{H}$ NMR spectral studies. The potent antibacterial and entomological (antifeedant, acaricidal, contact toxicity and stomach toxicity) activities of the synthesized compounds were investigated.
\end{abstract}

Key Words: Benzothiazole, Benzimidazole, Imidazoline, Antibacterial activity, Entomological activity

\section{Introduction}

Benzothiazole derivatives have been studied extensively and found to have diverse chemical reactivity and broad spectrum of biological activity such as antitumor agents, ${ }^{1-3}$ antimicrobial, $^{4-6}$ analgesics, ${ }^{7}$ anti-inflammatory, ${ }^{7-9}$ anti HIV, ${ }^{10}$ antileishmanial, ${ }^{11}$ etc. Further a wide range of therapeutic activities of benzimidazole show that they are also anticancer, ${ }^{12}$ antihypertension, ${ }^{13}$ antioxidant, ${ }^{14}$ antiviral, ${ }^{15}$ proton pump blocker, ${ }^{16}$ antinociceptive, ${ }^{17}$ anti-inflammatory, ${ }^{17,18}$ analgesic, ${ }^{18}$ antiparasites, ${ }^{19}$ human cytomegalovirus (HCMV) replication inhibitor, ${ }^{20}$ fungicidal, ${ }^{21}$ antihistamines, ${ }^{22,23}$ etc. Similarly Imidazoline derivatives also possess an array of biological activities. They are useful as anticancer, ${ }^{24,25}$ anti-inflammatory, ${ }^{25}$ Anthelmintic, ${ }^{26}$ antimicrobial, ${ }^{27}$ hypnotic agents, ${ }^{28}$ antiviral, ${ }^{29}$ anticoagulant, ${ }^{30}$ etc. These reports prompted us for synthesis of some new benzothiazole derivatives containing benzimidazole and imidazoline moiety. Substituted 2-aminobenzothiazole (1) was prepared by

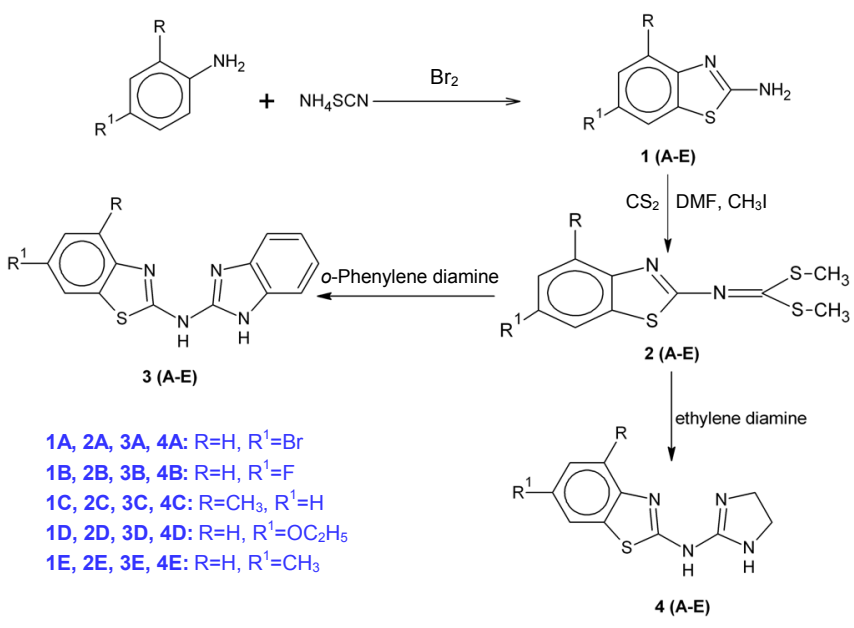

Scheme 1 thiocyanogenation of substituted anilines by the method reported earlier. ${ }^{31,32}$ These were reacted with carbon disulphide and methyl iodide in presence of con. $\mathrm{NaOH}$ aqueous solution leading to the formation of dimethyl (substituted-1,3-benzothiazol2-yl) dithioimidocarbonate derivative (2) which was treatment with bisnucleophiles viz. $o$-phenylene diamine and ethylene diamine gave $N$-(1H-benzimidazol-2-yl)-6-substituted-1,3benzothiazol-2-amine (3) and 6-substituted- $N$-(4,5-dihydro$1 H$-imidazol-2-yl)-1,3-benzothiazol-2-amine (4) (Scheme 1). The synthesized compounds were characterized by their elemental analysis, IR and ${ }^{1} \mathrm{H}$ NMR spectral studies. Antibacterial and entomological studies have been screened to observe their various biological activities.

\section{Experimental}

Reagent grade chemicals were used without further purification. Melting points were taken in open capillary tubes are uncorrected. The purity of the synthesized compounds was checked by Thin Layer Chromatographic studies. IR spectra were scanned on FT IR Perkins Elmer (Spectrum RX1) spectrophotometer $\left(\mathrm{cm}^{-1}\right)$ using a $\mathrm{KBr}$ disc. ${ }^{1} \mathrm{H}$ NMR spectra was recorded in tetramethylsilane (TMS) as the internal standard at $300 \mathrm{MHz}$ on a Bruker DRTX-300 spectrophotometer.

General Procedure for the Synthesis of 4/6-Substituted Benzimidazole.

Synthesis of Substituted-2-aminobenzothiazole Derivatives (1): Substituted-2-aminobenzothiazoles (1) were prepared as reported earlier. ${ }^{31,32}$

Synthesis of Dimethyl(substituted-1,3-benzothiazol-2-yl)dithioimidocarbonate Derivatives (2): To a well stirred ice cold solution of (1) (0.025 mole) in dimethylformamide $(10 \mathrm{~mL})$, were added $10 \mathrm{M} \mathrm{NaOH}$ aqueous solution $(4 \mathrm{~mL})$, carbon disulphide (0.05 mole) and methyl iodide $(0.025$ mole $)$ in sequence at an interval of $30 \mathrm{~min}$. and stirring was continued for $3 \mathrm{hrs}$. The mixture was then poured in ice cold water and the 
Table 1. Physical and chemical data of synthesized compounds

\begin{tabular}{ccccccc}
\hline $\begin{array}{c}\text { Com- } \\
\text { pound }\end{array}$ & $\mathrm{R}$ & $\mathrm{R}^{1}$ & Mol. Formula & $\begin{array}{c}\text { Mol. } \\
\text { Weight }\end{array}$ & $\begin{array}{c}\text { Melting } \\
\text { point }\left({ }^{\circ} \mathrm{C}\right)\end{array}$ & $\begin{array}{c}\text { Yield } \\
(\%)\end{array}$ \\
\hline 1A & $\mathrm{H}$ & $\mathrm{Br}$ & $\mathrm{C}_{7} \mathrm{H}_{5} \mathrm{BrN}_{2} \mathrm{~S}$ & 229.097 & $190-194$ & 70 \\
1B & $\mathrm{H}$ & $\mathrm{F}$ & $\mathrm{C}_{7} \mathrm{H}_{5} \mathrm{FN}_{2} \mathrm{~S}$ & 168.191 & $183-185$ & 68 \\
1C & $\mathrm{CH}_{3}$ & $\mathrm{H}$ & $\mathrm{C}_{8} \mathrm{H}_{8} \mathrm{~N}_{2} \mathrm{~S}$ & 164.227 & $137-139$ & 58 \\
1D & $\mathrm{H}$ & $\mathrm{OC}_{2} \mathrm{H}_{5}$ & $\mathrm{C}_{9} \mathrm{H}_{10} \mathrm{ON}_{2} \mathrm{~S}$ & 194.253 & $161-163$ & 72 \\
1E & $\mathrm{H}$ & $\mathrm{CH}_{3}$ & $\mathrm{C}_{8} \mathrm{H}_{8} \mathrm{~N}_{2} \mathrm{~S}$ & 164.227 & $140-143$ & 55 \\
2A & $\mathrm{H}$ & $\mathrm{Br}$ & $\mathrm{C}_{10} \mathrm{H}_{9} \mathrm{Br} \mathrm{N}_{2} \mathrm{~S}_{3}$ & 333.290 & $269-271$ & 70 \\
2B & $\mathrm{H}$ & $\mathrm{F}$ & $\mathrm{C}_{10} \mathrm{H}_{9} \mathrm{~F} \mathrm{~N}_{2} \mathrm{~S}_{3}$ & 272.391 & $190-194$ & 65 \\
2C & $\mathrm{CH}$ & $\mathrm{H}$ & $\mathrm{C}_{11} \mathrm{H}_{12} \mathrm{~N}_{2} \mathrm{~S}_{3}$ & 268.427 & $104-106$ & 58 \\
2D & $\mathrm{H}$ & $\mathrm{OC}_{2} \mathrm{H}_{5}$ & $\mathrm{C}_{12} \mathrm{H}_{14} \mathrm{ON}_{2} \mathrm{~S}_{3}$ & 298.447 & $187-191$ & 80 \\
2E & $\mathrm{H}$ & $\mathrm{CH}$ & $\mathrm{C}_{11} \mathrm{H}_{12} \mathrm{~N}_{2} \mathrm{~S}_{3}$ & 268.427 & $109-112$ & 59 \\
3A & $\mathrm{H}$ & $\mathrm{Br}$ & $\mathrm{C}_{14} \mathrm{H}_{9} \mathrm{BrN}_{4} \mathrm{~S}$ & 345.217 & $274-277$ & 60 \\
3B & $\mathrm{H}$ & $\mathrm{F}$ & $\mathrm{C}_{14} \mathrm{H}_{9} \mathrm{FN}_{4} \mathrm{~S}$ & 284.311 & $259-262$ & 52 \\
3C & $\mathrm{CH}$ & $\mathrm{H}$ & $\mathrm{C}_{15} \mathrm{H}_{12} \mathrm{~N}_{4} \mathrm{~S}$ & 280.347 & $256-260$ & 48 \\
3D & $\mathrm{H}$ & $\mathrm{OC}_{2} \mathrm{H}_{5}$ & $\mathrm{C}_{16} \mathrm{H}_{14} \mathrm{ON}_{4} \mathrm{~S}$ & 310.373 & $269-272$ & 68 \\
3E & $\mathrm{H}$ & $\mathrm{CH}_{3}$ & $\mathrm{C}_{15} \mathrm{H}_{12} \mathrm{~N}_{4} \mathrm{~S}$ & 280.347 & $262-265$ & 50 \\
4A & $\mathrm{H}$ & $\mathrm{Br}^{2}$ & $\mathrm{C}_{10} \mathrm{H}_{9} \mathrm{BrN}_{4} \mathrm{~S}$ & 297.174 & $220-223$ & 82 \\
4B & $\mathrm{H}$ & $\mathrm{F}$ & $\mathrm{C}_{10} \mathrm{H}_{9} \mathrm{FN}_{4} \mathrm{~S}$ & 236.268 & $224-227$ & 75 \\
4C & $\mathrm{CH}$ & $\mathrm{H}$ & $\mathrm{C}_{11} \mathrm{H}_{12} \mathrm{~N}_{4} \mathrm{~S}$ & 232.304 & $259-262$ & 58 \\
4D & $\mathrm{H}$ & $\mathrm{OC}_{2} \mathrm{H}_{5}$ & $\mathrm{C}_{12} \mathrm{H}_{14} \mathrm{ON}_{4} \mathrm{~S}$ & 262.330 & $204-206$ & 65 \\
4E & $\mathrm{H}$ & $\mathrm{CH}_{3}$ & $\mathrm{C}_{11} \mathrm{H}_{12} \mathrm{~N}_{4} \mathrm{~S}$ & 232.304 & $236-240$ & 65 \\
\hline
\end{tabular}

resulting solid was washed with water and recrystallised from aq. ethanol. Physical and chemical data of synthesized compounds are given in Table 1.

Dimethyl (6-Bromo-1,3-benzothiazol-2-yl) Dithioimidocarbonate Derivatives (2A) - Yield 70\%. Anal. Calcd for $\mathrm{C}_{10} \mathrm{H}_{9}$ $\mathrm{BrN}_{2} \mathrm{~S}_{3}$ : C - 36.04\%, N - 8.41\%, H - 2.72\%, Found C - 36.14\%, $\mathrm{N}-8.45 \%$, H - 2.78\%; IR (KBr) 3069, 1599, 1502, 1172, 1081, 809, 753 and 635 (benzothiazole with aromatic ring), 2880 (aliphatic $\mathrm{CH}), 1632(\mathrm{C}=\mathrm{N}), 1263,1310(\mathrm{C}-\mathrm{S}), 561(\mathrm{C}-\mathrm{Br}),{ }^{1} \mathrm{H}$ NMR (300 MHz, DMSO- $\left.d_{6}\right): 2.65$ (s, $\left.6 \mathrm{H}, 2 \times \mathrm{SCH}_{2}\right) ; 6.98-7.38$ (m, 3H, Ar-H).

Dimethyl (6-Fluoro-1,3-benzothiazol-2-yl) Dithioimidocarbonate Derivatives (2B) - Yield 65\%. Anal. Calcd for $\mathrm{C}_{10} \mathrm{H}_{9}$ $\mathrm{FN}_{2} \mathrm{~S}_{3}: \mathrm{C}-44.09 \%, \mathrm{~N}-10.28 \%, \mathrm{H}-3.33 \%$, Found C - $44.05 \%$, N - 10.25\%, H - 3.28\%; IR (KBr) 3050, 1549, 1506, 1161, 1047 , 837, 745 and 631 (benzothiazole with aromatic ring), 2905 (aliphatic $\mathrm{CH}), 1628(\mathrm{C}=\mathrm{N}), 1309,1244(\mathrm{C}-\mathrm{S}), 1005(\mathrm{C}-\mathrm{F}),{ }^{1} \mathrm{H}$ NMR (300 MHz, DMSO- $\left.d_{6}\right): 2.68\left(\mathrm{~s}, 6 \mathrm{H}, 2 \times \mathrm{SCH}_{2}\right) ; 7.08-7.29$ (m, 3H, Ar-H).

Dimethyl (4-Methyl-1,3-benzothiazol-2-yl) Dithioimidocarbonate Derivatives (2C) - Yield 58\%. Anal. Calcd for $\mathrm{C}_{11} \mathrm{H}_{12}$ $\mathrm{N}_{2} \mathrm{~S}_{3}$ : C - 49.22\%, N - 10.44\%, H - 4.51\%, Found C - 49.20\%, $\mathrm{N}-10.39 \%$, H - 4.45\%; IR (KBr) 3077, 1601, 1455, 1163, 1081, 840, 735 and 658 (benzothiazole with aromatic ring), 2895 (aliphatic $\mathrm{CH}), 1635(\mathrm{C}=\mathrm{N}), 1312,1260(\mathrm{C}-\mathrm{S}) .{ }^{1} \mathrm{H} \mathrm{NMR}(300 \mathrm{MHz}$, DMSO-d $\left.d_{6}\right): 2.58\left(\mathrm{~s}, 6 \mathrm{H}, 2 \times \mathrm{SCH}_{2}\right) ; 2.45\left(\mathrm{~s}, 3 \mathrm{H}, \mathrm{Ar} \mathrm{CH}_{3}\right), 7.12-$ $7.53(\mathrm{~m}, 3 \mathrm{H}, \mathrm{Ar}-\mathrm{H})$.

Dimethyl (6-Ethoxy-1,3-benzothiazol-2-yl) Dithioimidocarbonate Derivatives (2D) - Yield $80 \%$. Anal. Calcd for $\mathrm{C}_{12} \mathrm{H}_{14}$ $\mathrm{ON}_{2} \mathrm{~S}_{3}$ : C - 48.29\%, N - 9.39\%, H - 4.73\%, Found C - 48.35, N - 9.45\%, H - 4.79\%; IR (KBr) 3095, 1605, 1482, 1178, 1020, 832,735 and 675 (benzothiazole with aromatic ring), 2892 (aliphatic $\mathrm{CH}), 1632(\mathrm{C}=\mathrm{N}), 1366,1221(\mathrm{C}-\mathrm{S}), 1260(\mathrm{C}-\mathrm{O}-\mathrm{C}),{ }^{1} \mathrm{H}$
NMR (300 MHz, DMSO- $\left.d_{6}\right): 1.52\left(\mathrm{t}, 3 \mathrm{H}, \mathrm{OCH}_{2} \mathrm{CH}_{3}\right) ; 2.52(\mathrm{~s}$, $6 \mathrm{H}, 2 \times \mathrm{SCH}_{2}$ ); 4.02 (q, 2H, $\mathrm{Ar} \mathrm{OCH}$ ), 7.14-7.43 (m, 3H, Ar-H).

Dimethyl (6-Methyl-1,3-benzothiazol-2-yl) Dithioimidocarbonate Derivatives (2E) - Yield 58\%. Anal. Calcd for $\mathrm{C}_{11} \mathrm{H}_{12}$ $\mathrm{N}_{2} \mathrm{~S}_{3}$ : C - 49.22\%, N - 10.44\%, H - 4.51\%, Found C - 49.19\%, $\mathrm{N}-10.40 \%$, H - 4.58\%; IR (KBr) 3086, 1605, 1464, 1160, 1085, 835, 732 and 665 (benzothiazole with aromatic ring), 2895 (aliphatic $\mathrm{CH}), 1635(\mathrm{C}=\mathrm{N}), 1312,1260(\mathrm{C}-\mathrm{S}) .{ }^{1} \mathrm{H} \mathrm{NMR}(300 \mathrm{MHz}$, DMSO-d $\left.d_{6}\right): 2.62\left(\mathrm{~s}, 6 \mathrm{H}, 2 \times \mathrm{SCH}_{2}\right) ; 2.41\left(\mathrm{~s}, 3 \mathrm{H}, \mathrm{ArCH}_{3}\right), 7.20-$ 7.49 (m, 3H, Ar-H).

Synthesis of $\mathrm{N}$-(1H-Benzimidazol-2-yl)-substituted-1,3benzothiazol-2-amine Derivatives (3): To a solution of 2 (5.0 $\mathrm{mmol})$ in DMF $(20 \mathrm{~mL})$ was added in solution of o-phenylene diamine (4.0 mmole) in DMF $(15 \mathrm{~mL})$ with stirring at room temp. The reaction mixture was reflux for $8 \mathrm{hrs}$. The mixture was poured on crushed ice. The resulting solid was dried and recrystallised from ethanol. Physical and chemical data of synthesized compounds are summarized in Table 1.

$\mathrm{N}$-(1H-Benzimidazol-2-yl)-6-bromo-1,3-benzothiazol-2-a mine (3A) - Yield 60\%. Anal. Calcd for $\mathrm{C}_{14} \mathrm{H}_{9} \mathrm{BrN}_{4} \mathrm{~S}$ : C $48.71 \%$, N - 16.23\%, H - 2.63\%, Found C - 48.68, N - 16.20\%, $\mathrm{H}-2.61 \%$; IR (KBr) 3072, 1540, 1470, 1150, 1051, 810, 743 and 640 (benzothiazole with aromatic ring), $3369(\mathrm{~N}-\mathrm{H}), 1635$ $(\mathrm{C}=\mathrm{N}), 1308$ (C-S), 590 (C-Br), ${ }^{1} \mathrm{HNMR}\left(300 \mathrm{MHz}, \mathrm{DMSO}-d_{6}\right)$ : $6.10(\mathrm{~s}, 1 \mathrm{H}$, acyclic NH); 7.10-7.43 (m, 7H, Ar-H); 8.04 (s, 1H, cyclic NH).

$\mathrm{N}$-(1 H-Benzimidazol-2-yl)-6-fluoro-1,3-benzothiazol-2amine (3B) - Yield 52\%. Anal. Calcd for $\mathrm{C}_{14} \mathrm{H}_{9} \mathrm{FN}_{4} \mathrm{~S}: \mathrm{C}-$ $59.14 \%, \mathrm{~N}-19.71 \%$, H - 3.19\%, Found C - 54.18, N - 19.75\%, H - 3.21\%; IR (KBr) 3068, 1568, 1478, 1108, 1068, 808, 740 and 658 (benzothiazole with aromatic ring), $3355(\mathrm{~N}-\mathrm{H}), 1638$ $(\mathrm{C}=\mathrm{N}), 1315$ (C-S), 1008 (C-F), ${ }^{1} \mathrm{HNMR}$ (300 MHz, DMSO- $\left.d_{6}\right)$ : 5.74 (s, 1H, acyclic NH); 6.91-7.33 (m, 7H, Ar-H); 8.07 (s, 1H, cyclic $\mathrm{NH})$.

$\mathrm{N}$-(1 H-Benzimidazol-2-yl)-4-methyl-1,3-benzothiazol-2amine (3C) - Yield 48\%. Anal. Calcd for $\mathrm{C}_{15} \mathrm{H}_{12} \mathrm{~N}_{4} \mathrm{~S}: \mathrm{C}-64.26 \%$, N - 19.98\%, H - 4.31\%, Found C - 64.28, N - 19.97\%, H - 4.35\%; IR (KBr) 3087, 1603, 1462, 1156, 1073, 841, 728 and 683 (benzothiazole with aromatic ring), $3357(\mathrm{~N}-\mathrm{H}), 1644(\mathrm{C}=\mathrm{N})$, $2887(\mathrm{CH}), 1304$ (C-S), ${ }^{1} \mathrm{HNMR}$ (300 MHz, DMSO- $\left.d_{6}\right): 2.50$ (s, 3H, $\left.\mathrm{CH}_{3}\right) ; 5.89$ (s, $1 \mathrm{H}$, acyclic $\left.\mathrm{NH}\right)$; 6.98-7.45 (m, 7H, Ar-H); 7.98 (s, 1H, cyclic NH).

$\mathrm{N}$-(1H-Benzimidazol-2-yl)-6-ethoxy-1,3-benzothiazol-2amine (3D) - Yield 68\%. Anal. Calcd for $\mathrm{C}_{16} \mathrm{H}_{14} \mathrm{ON}_{4} \mathrm{~S}$ : C $61.92 \%, \mathrm{~N}-18.05 \%$, H - 4.55\%, Found C - 61.98, N - 18.07\%, $\mathrm{H}-4.59 \%$; IR (KBr) 3085, 1599, 1456, 1150, 1038, 830, 728 and 672 (benzothiazole with aromatic ring), $3345(\mathrm{~N}-\mathrm{H}), 1628$ $(\mathrm{C}=\mathrm{N}), 1363$ (C-S), 1261 (C-O-C), 2905 (CH), ${ }^{1} \mathrm{HNMR}$ (300 $\left.\mathrm{MHz}, \mathrm{DMSO}-d_{6}\right): 1.39$ (t, $\left.3 \mathrm{H}, \mathrm{OCH}_{2} \mathrm{CH}_{3}\right) ; 3.98$ (q, 2H, Ar$\left.\mathrm{OCH}_{2}\right), 5.80(\mathrm{~s}, 1 \mathrm{H}$, acyclic $\mathrm{NH}) ; 7.09-7.68$ (m, 7H, Ar- H); 8.12 (s, $1 \mathrm{H}$, cyclic $\mathrm{NH})$.

$\mathrm{N}$-(1H-Benzimidazol-2-yl)-6-methyl-1,3-benzothiazol-2amine (3E) - Yield 50\%. Anal. Calcd for $\mathrm{C}_{15} \mathrm{H}_{12} \mathrm{~N}_{4} \mathrm{~S}$ : C - 64.26\%, $\mathrm{N}-19.98 \%, \mathrm{H}-4.31 \%$, Found C - 64.24, N - 20.05\%, H $4.36 \%$; IR (KBr) 3085, 1600, 1460, 1155, 1080, 835, 731 and 687 (benzothiazole with aromatic ring), $3364(\mathrm{~N}-\mathrm{H}), 2908(\mathrm{CH})$, $1639(\mathrm{C}=\mathrm{N}), 1308(\mathrm{C}-\mathrm{S}),{ }^{1} \mathrm{HNMR}\left(300 \mathrm{MHz}, \mathrm{DMSO}-d_{6}\right): 2.48$ (s, $\left.3 \mathrm{H}, \mathrm{CH}_{3}\right) ; 5.79$ (s, $1 \mathrm{H}$, acyclic $\left.\mathrm{NH}\right)$; 6.97-7.36 (m, 7H, Ar-H); 
Table 2. The zone of inhibition in mm of the compound as well as standard drugs tested for antibacterial activity

\begin{tabular}{|c|c|c|c|c|c|c|c|c|c|c|}
\hline \multirow{2}{*}{ Compound } & \multirow{2}{*}{$\mathrm{R}$} & \multirow{2}{*}{$\mathrm{R}^{1}$} & \multicolumn{2}{|c|}{ E. coli } & \multicolumn{2}{|c|}{ K. species } & \multicolumn{2}{|c|}{ M. luteus } & \multicolumn{2}{|c|}{ S. aureus } \\
\hline & & & $200 \mu \mathrm{g} / \mathrm{ml}$ & $100 \mu \mathrm{g} / \mathrm{mL}$ & $200 \mu \mathrm{g} / \mathrm{mL}$ & $100 \mu \mathrm{g} / \mathrm{mL}$ & $200 \mu \mathrm{g} / \mathrm{mL}$ & $100 \mu \mathrm{g} / \mathrm{mL}$ & $200 \mu \mathrm{g} / \mathrm{mL}$ & $100 \mu \mathrm{g} / \mathrm{mL}$ \\
\hline $2 A$ & $\mathrm{H}$ & $\mathrm{Br}$ & 12 & 10 & 09 & 06 & 11 & 09 & 11 & 09 \\
\hline $2 B$ & $\mathrm{H}$ & $\mathrm{F}$ & 11 & 10 & 10 & 08 & 10 & 08 & 09 & 07 \\
\hline $2 \mathrm{C}$ & $\mathrm{CH}_{3}$ & $\mathrm{H}$ & 11 & 09 & 08 & 00 & 00 & 00 & 08 & 07 \\
\hline 2D & $\mathrm{H}$ & $\mathrm{OC}_{2} \mathrm{H}_{5}$ & 12 & 03 & 06 & 00 & 08 & 07 & 10 & 08 \\
\hline $2 \mathrm{E}$ & $\mathrm{H}$ & $\mathrm{CH}_{3}$ & 10 & 08 & 07 & 00 & 00 & 00 & 06 & 00 \\
\hline 3A & $\mathrm{H}$ & $\mathrm{Br}$ & 18 & 16 & 09 & 08 & 18 & 16 & 16 & 13 \\
\hline 3B & $\mathrm{H}$ & $\mathrm{F}$ & 16 & 14 & 11 & 10 & 16 & 14 & 12 & 10 \\
\hline $3 C$ & $\mathrm{CH}_{3}$ & $\mathrm{H}$ & 11 & 09 & 08 & 07 & 10 & 06 & 10 & 07 \\
\hline 3D & $\mathrm{H}$ & $\mathrm{OC}_{2} \mathrm{H}_{5}$ & 14 & 11 & 07 & 06 & 09 & 08 & 14 & 12 \\
\hline $3 \mathbf{E}$ & $\mathrm{H}$ & $\mathrm{CH}_{3}$ & 11 & 09 & 08 & 06 & 09 & 06 & 08 & 00 \\
\hline $4 A$ & $\mathrm{H}$ & $\mathrm{Br}$ & 21 & 19 & 11 & 08 & 21 & 19 & 17 & 15 \\
\hline $4 B$ & $\mathrm{H}$ & $\mathrm{F}$ & 18 & 16 & 14 & 10 & 19 & 17 & 13 & 10 \\
\hline $4 C$ & $\mathrm{CH}_{3}$ & $\mathrm{H}$ & 13 & 11 & 09 & 07 & 09 & 08 & 11 & 09 \\
\hline 4D & $\mathrm{H}$ & $\mathrm{OC}_{2} \mathrm{H}_{5}$ & 16 & 13 & 08 & 06 & 10 & 08 & 16 & 13 \\
\hline $4 E$ & $\mathrm{H}$ & $\mathrm{CH}_{3}$ & 11 & 10 & 09 & 06 & 09 & 08 & 08 & 00 \\
\hline DMF & & & & 00 & & 00 & & 00 & & 00 \\
\hline $\begin{array}{l}\text { Novobiocin } \\
100 \mu \mathrm{g} / \mathrm{mL}\end{array}$ & & & & 18 & & 07 & & 35 & & 15 \\
\hline $\begin{array}{l}\text { Kanamycin } \\
100 \mu \mathrm{g} / \mathrm{mL}\end{array}$ & & & & 28 & & 07 & & 25 & & 18 \\
\hline $\begin{array}{l}\text { Amikacin } \\
100 \mu \mathrm{g} / \mathrm{mL}\end{array}$ & & -- & & 22 & & 16 & & 32 & & 18 \\
\hline
\end{tabular}

8.02 (s, 1H, cyclic NH).

Synthesis of Substituted- $\mathrm{N}$-(4,5-dihydro- $1 H$-imidazol-2-yl)1,3-benzothiazol-2-amine Derivatives (4): To a solution of 2 (4.0 mmole) in DMF (15 mL) was added in solution of ethylene diamine $(8.0 \mathrm{mmole})$ in DMF $(15 \mathrm{~mL})$ with stirring at room temp. The reaction mixture was refluxed for $8 \mathrm{hrs}$. The mixture was poured on crushed ice. The resulting solid was dried and recrystallised from ethanol. Physical and chemical data of synthesized compounds are given in Table 1.

6-Bromo- $N$-(4,5-dihydro-1 $H$-imidazol-2-yl)-1,3-benzothiazol-2-amine (4A) - Yield 82\%. Anal. Calcd for $\mathrm{C}_{10} \mathrm{H}_{9} \mathrm{BrN}_{4} \mathrm{~S}$ : C - 40.42\%, H - 3.05\%, N - 18.85\%, Found C - 41.35\%, H $3.08 \%$, N - 18.76\%; IR (KBr) 3070, 1599, 1471, 1100, 1075, 806,733 and 659 (benzothiazole with aromatic ring), 3351 (N-H), $1637(\mathrm{C}=\mathrm{N}), 1304$ (C-S), 603 (C-Br), ${ }^{1} \mathrm{HNMR}(300 \mathrm{MHz}$, DMSO-d $\left.)_{6}\right): 3.58$ (s, 4H, $\left.2 \times \mathrm{CH}_{2}\right), 5.68(\mathrm{~s}, 1 \mathrm{H}$, acyclic $\mathrm{N}-\mathrm{H})$, 7.18-7.61 (m, 3H, Ar-H), 8.14 (s, 1H, cyclic N-H).

6-Fluoro- $\mathrm{N}$-(4,5-dihydro-1 $\mathrm{H}$-imidazol-2-yl)-1,3-benzothiazol-2-amine (4B) - Y ield 75\%. Anal. Calcd for $\mathrm{C}_{10} \mathrm{H}_{9} \mathrm{FN}_{4} \mathrm{~S}$ : $\mathrm{C}-50.83 \%, \mathrm{H}-3.84 \%, \mathrm{~N}-23.71 \%$, Found C - 50.81\%, H $3.80 \%$, N - 23.65\%; IR (KBr) 3047, 1570, 1498, 1099, 1051, 820, 737 and 644 (benzothiazole with aromatic ring), 3305 $(\mathrm{N}-\mathrm{H}), 1630(\mathrm{C}=\mathrm{N}), 1307$ (C-S), $1010(\mathrm{C}-\mathrm{F}),{ }^{1} \mathrm{HNMR}(300 \mathrm{MHz}$, DMSO- $\left.d_{6}\right): 3.56\left(\mathrm{~s}, 4 \mathrm{H}, 2 \times \mathrm{CH}_{2}\right), 5.60(\mathrm{~s}, 1 \mathrm{H}$, acyclic $\mathrm{N}-\mathrm{H})$, 7.16-7.58 (m, 3H, Ar-H), 8.09 (s, 1H, cyclic N-H).

4-Methyl- $\mathrm{N}$-(4,5-dihydro-1 $\mathrm{H}$-imidazol-2-yl)-1,3-benzothiazol-2-amine (4C) - Yield 58\%. Anal. Calcd for $\mathrm{C}_{11} \mathrm{H}_{12} \mathrm{~N}_{4} \mathrm{~S}$ : $\mathrm{C}-56.87 \%, \mathrm{H}-5.21 \%, \mathrm{~N}-24.12 \%$, Found C - $57.03 \%, \mathrm{H}-$ $5.25 \%, \mathrm{~N}-24.18 \%$; IR (KBr) 3090, 1610, 1454, 1162, 1102 , 832,718 and 655 (benzothiazole with aromatic ring), 3398 $(\mathrm{N}-\mathrm{H}), 1640(\mathrm{C}=\mathrm{N}), 1320(\mathrm{C}-\mathrm{S}), 2857(\mathrm{CH}),{ }^{1} \mathrm{H} \mathrm{NMR}(300 \mathrm{MHz}$,
DMSO- $\left.d_{6}\right): 3.58\left(\mathrm{~s}, 4 \mathrm{H}, 2 \times \mathrm{CH}_{2}\right), 2.52\left(\mathrm{~s}, 3 \mathrm{H}, \mathrm{Ar}-\mathrm{CH}_{3}\right), 5.70$ (s, 1H, acyclic N-H), 6.99-7.42 (m, 3H, Ar-H), $8.18(\mathrm{~s}, 1 \mathrm{H}$, cyclic N-H).

6-Ethoxy- $\mathrm{N}$-(4,5-dihydro-1H-imidazol-2-yl)-1,3-benzothiazol-2-amine (4D) - Yield 65\%. Anal. Calcd for $\mathrm{C}_{12} \mathrm{H}_{14} \mathrm{ON}_{4} \mathrm{~S}$ : C - 54.94\%, H - 5.38\%, N - 21.36\%, Found C - 55.01\%, H $5.47 \%$, N - 21.39\%; IR (KBr) 3095, 1489, 1452, 1113, 1057 , 820,723 and 671 (benzothiazole with aromatic ring), 3316 $(\mathrm{N}-\mathrm{H}), 1620(\mathrm{C}=\mathrm{N}), 1360$ (C-S), 1265 (C-O-C), 2898 (CH), ${ }^{1} \mathrm{HNMR}\left(300 \mathrm{MHz}, \mathrm{DMSO}-d_{6}\right): 1.38\left(\mathrm{t}, 3 \mathrm{H}, \mathrm{CH}_{3}\right), 3.30$ (s, $\left.4 \mathrm{H}, 2 \times \mathrm{CH}_{2}\right), 4.04$ (q, 2H, Ar- $\left.\mathrm{OCH}_{2}\right), 6.08(\mathrm{~s}, \mathrm{H}$, acyclic $\mathrm{NH})$, 7.18-7.44 (m, 3H, Ar-H), 7.98 (s, H, cyclic NH).

6-Methyl- $\mathrm{N}$-(4,5-dihydro-1 $\mathrm{H}$-imidazol-2-yl)-1,3-benzothiazol-2-amine (4E) - Yield 65\%. Anal. Calcd for $\mathrm{C}_{11} \mathrm{H}_{12} \mathrm{~N}_{4} \mathrm{~S}$ : C $56.87 \%$, H - 5.21\%, N - 24.12\%, Found C - $57.03 \%$, H - 5.28\%, $\mathrm{N}-24.22 \%$; IR (KBr) 3085, 1605, 1450, 1158, 1100, 832, 715 and 653 (benzothiazole with aromatic ring), $3382(\mathrm{~N}-\mathrm{H}), 1635$ $(\mathrm{C}=\mathrm{N}), 1315(\mathrm{C}-\mathrm{S}), 2853(\mathrm{CH}),{ }^{1} \mathrm{H}$ NMR (300 MHz, DMSO- $\left.d_{6}\right)$ : $3.53\left(\mathrm{~s}, 4 \mathrm{H}, 2 \times \mathrm{CH}_{2}\right), 2.51\left(\mathrm{~s}, 3 \mathrm{H}, \mathrm{Ar}-\mathrm{CH}_{3}\right), 5.68(\mathrm{~s}, 1 \mathrm{H}$, acyclic $\mathrm{N}-\mathrm{H}), 6.95-7.35$ (m, 3H, Ar-H), 8.10 (s, 1H, cyclic N-H).

Antibacterial Activity. All the synthesized compounds were tested against gram positive bacteria Staphylococcus aureus and Micrococcus luteus and gram negative bacteria Escherichia coli, and Klebsiella species using paper disc method. ${ }^{33}$ Muller Hinton Agar (Hi-Media Pvt. Ltd. Mumbai, India) was used to culture the test bacteria. The microbial culture were grown at $37^{\circ} \mathrm{C}$ for 8 hours and then appropriately diluted with sterile $0.8 \%$ saline solution. The concentration of test drugs was kept $100 \mu \mathrm{g} / \mathrm{mL}$ in DMF. Standard drugs Novobiocin, Kanamycin and Amikacin were used for comparison. The antimicrobial activity was evaluated by measuring the zones of growth inhibi- 
Table 3. Antifeedant Activity

\begin{tabular}{cccccc}
\hline \multirow{2}{*}{ S. No. Compounds } & $\begin{array}{c}\text { Fiducial } \\
\text { Limits }\end{array}$ & Slope \pm & $\begin{array}{c}\text { Chi. Sq. } \\
\text { (3) }\end{array}$ & $\begin{array}{c}\mathrm{LC}_{50} / \mathrm{LD}_{50} \\
\text { At } 24 \mathrm{hrs}\end{array}$ \\
\hline 1 & 3A & $0.25-0.40$ & $1.17 \pm 0.14$ & $7.23(3)$ & 0.31 \\
2 & 3B & $0.64-1.16$ & $1.47 \pm 0.16$ & $3.73(3)$ & 0.83 \\
3 & 3C & $0.45-1.09$ & $0.87 \pm 0.13$ & $1.71(3)$ & 0.64 \\
4 & 3D & $0.29-0.51$ & $1.01 \pm 0.13$ & $5.39(3)$ & 0.37 \\
5 & 3E & $0.37-0.63$ & $1.18 \pm 0.14$ & $2.36(3)$ & 0.47 \\
6 & 4A & $0.30-0.59$ & $0.93 \pm 0.13$ & $2.29(3)$ & 0.41 \\
7 & 4B & $0.51-0.97$ & $1.21 \pm 0.14$ & $2.25(3)$ & 0.67 \\
8 & 4C & $0.84-2.34$ & $1.06 \pm 0.15$ & $0.70(3)$ & 1.24 \\
9 & 4D & $0.43-0.87$ & $1.03 \pm 0.14$ & $0.34(3)$ & 0.58 \\
10 & 4E & $0.43-0.72$ & $1.31 \pm 0.14$ & $0.59(3)$ & 0.54 \\
\hline
\end{tabular}

Table 4. Acaricidal Activity

\begin{tabular}{cccccc}
\hline \multirow{2}{*}{ S. No. Compounds } & $\begin{array}{c}\text { Fiducial } \\
\text { Limits }\end{array}$ & Slope \pm & $\begin{array}{c}\text { Chi. Sq. } \\
\text { (3) }\end{array}$ & $\begin{array}{c}\mathrm{LC}_{50} / \mathrm{LD}_{50} \\
\text { At } 24 \mathrm{hrs} .\end{array}$ \\
\hline 1 & 3A & $0.14-0.31$ & $0.96 \pm 0.09$ & $7.52(3)$ & 0.20 \\
2 & 3B & $0.12-0.30$ & $0.80 \pm 0.08$ & $6.91(3)$ & 0.18 \\
3 & 3C & $0.06-0.10$ & $1.24 \pm 0.10$ & $14.27(3)$ & 0.08 \\
4 & 3D & $0.36-1.89$ & $0.64 \pm 0.08$ & $3.57(3)$ & 0.70 \\
5 & 3E & $0.07-0.22$ & $0.76 \pm 0.06$ & $5.63(3)$ & 0.14 \\
6 & 4A & $0.07-0.20$ & $0.64 \pm 0.06$ & $8.43(3)$ & 0.11 \\
7 & 4B & $0.08-0.17$ & $0.81 \pm 0.7$ & $9.10(3)$ & 0.11 \\
8 & 4C & $0.05-0.10$ & $0.87 \pm 0.7$ & $20.01(3)$ & 0.07 \\
9 & 4D & $0.04-0.09$ & $0.70 \pm 0.06$ & $4.61(3)$ & 0.05 \\
10 & 4E & $0.08-0.16$ & $0.98 \pm 0.08$ & $15.28(3)$ & 0.11 \\
\hline
\end{tabular}

tion around disc of test organism and results are given in Table 2.

Antifeedant Activity. The antifeedant activity of these compounds was also carried out by leaf dip method, ${ }^{34,35}$ using fourth instar larvae of Spodoptera litura. The leaf discs of about $25 \mathrm{~cm}^{2}$ were prepared and dipped for thirty seconds in various test compounds. The leaf discs were air dried to evaporate the excess acetone and then offered for feeding. The insects were allowed to feed for $24 \mathrm{hrs}$. After $24 \mathrm{hrs}$. leaf area uneaten was measured by using leaf area meter. The difference between leaf area provided and the leaf area uneaten is taken as amount of leaf area consumed. The feeding inhibition was calculated and used for calculation of effective concentration $\left(\mathrm{EC}_{50} / \mathrm{LD}_{50}\right)$ using Maximum likelihood programmer MLP 3.01. The results of antifeedant activity are summarized in Table 3.

Acaricidal Activity. The acaricidal activity of these compounds was carried out by leaf dip method. ${ }^{34,35}$ Leaf discs of Mulberry $\left(5 \mathrm{~cm}^{2}\right.$ diameter) were dipped in different compounds for 30 seconds. The leaf discs were air dried to remove the excess of acetone and placed over wet cotton in Petri plate. The adult female mites were released on treated leaf discs and mortality data were recorded after 24 hours. Mites released on leaf treated only with acetone and tween 20 emulsifier served as control. The mortality data was used for calculation of $\mathrm{LC}_{50} / \mathrm{LD}_{50}$ using Maximum Likelihood Programmer MLP 3.01. The results of acaricidal activity are summarized in Table 4.

Contact Toxicity. The contact toxicity of these compounds was carried out by topical application method ${ }^{36,37}$ against larvae of Spodoptera litura, which are harmful for Indian crops. First
Table 5. Contact Toxicity

\begin{tabular}{cccccc}
\hline S. No. Compounds & $\begin{array}{c}\text { Fiducial } \\
\text { Limits }\end{array}$ & Slope \pm & $\begin{array}{c}\text { Chi. Sq. } \\
(3)\end{array}$ & $\begin{array}{c}\mathrm{LC}_{50} / \mathrm{LD}_{50} \\
\text { At } 24 \mathrm{hrs}\end{array}$ \\
\hline 1 & 3A & $0.92-2.60$ & $11.11 \pm 0.16$ & $0.26(3)$ & 1.36 \\
2 & 3B & $0.41-0.61$ & $1.63 \pm 0.15$ & $1.84(3)$ & 0.49 \\
3 & 3C & $2.37-32.67$ & $0.95 \pm 0.19$ & $0.611(3)$ & 5.38 \\
4 & 3D & $0.45-0.68$ & $1.69 \pm 0.16$ & $1.22(3)$ & 0.55 \\
5 & 3E & $0.40-0.60$ & $1.58 \pm 0.15$ & $0.72(3)$ & 0.48 \\
6 & 4A & $0.02-0.27$ & $225 \pm 0.17$ & $4.13(3)$ & 0.23 \\
7 & 4B & $2.70-51.84$ & $0.96 \pm 0.20$ & $0.54(3)$ & 6.61 \\
8 & 4C & $0.48-0.75$ & $1.61 \pm 0.16$ & $2.94(3)$ & 0.59 \\
9 & 4D & $0.40-0.59$ & $1.66 \pm 0.15$ & $5.66(3)$ & 0.48 \\
10 & 4E & $0.88-1.83$ & $1.48 \pm 0.18$ & $1.41(3)$ & 1.18 \\
\hline
\end{tabular}

Table 6. Stomach Toxicity

\begin{tabular}{cccccc}
\hline \multirow{2}{*}{ S. No. Compounds } & $\begin{array}{c}\text { Fiducial } \\
\text { Limits }\end{array}$ & Slope \pm & $\begin{array}{c}\text { Chi. Sq. } \\
\text { (3) }\end{array}$ & $\begin{array}{c}\mathrm{LC}_{50} / \mathrm{LD}_{50} \\
\text { At } 24 \mathrm{hrs}\end{array}$ \\
\hline 1 & 3A & $1.69-7.75$ & $1.28 \pm 0.21$ & $0.38(3)$ & 2.89 \\
2 & 3B & $0.44-0.67$ & $1.65 \pm 0.16$ & $3.89(3)$ & 0.53 \\
3 & 3C & $1.88-14.44$ & $1.01 \pm 0.18$ & $1.06(3)$ & 3.70 \\
4 & 3D & $0.58-0.97$ & $1.54 \pm 0.16$ & $2.33(3)$ & 0.72 \\
5 & 3E & $0.44-0.67$ & $1.69 \pm 0.16$ & $3.30(3)$ & 0.53 \\
6 & 4A & $0.35-0.56$ & $1.40 \pm 0.14$ & $0.82(3)$ & 0.44 \\
7 & 4B & $0.44-0.73$ & $1.40 \pm 0.15$ & $3.84(3)$ & 0.55 \\
8 & 4C & $0.54-0.90$ & $1.49 \pm 0.16$ & $3.39(3)$ & 0.68 \\
9 & 4D & $0.57-1.05$ & $1.32 \pm 0.15$ & $0.63(3)$ & 0.74 \\
10 & 4E & $3.69-38.46$ & $0.77 \pm 0.19$ & $0.39(3)$ & 12.64 \\
\hline
\end{tabular}

the given compounds were dissolved in acetone and then each compound was applied on the dorsal surface of the larvae. About $10 \mu \mathrm{L}$ of each concentration was applied on each larva. Some of the larvae of insect treated by acetone alone, worked as control. Now the mortality data was recorded after $24 \mathrm{hrs,}$ and the treated mortality was corrected with control morality. These corrected mortality data was used for calculation of $\mathrm{LC}_{50} /$ $\mathrm{LD}_{50}$ using Maximum Likelihood programmer MLP 3.01. The results of Contact Toxicity are summarized in Table 5.

Stomach Toxicity. The stomach toxicity of these compounds was carried out by leaf dip method. ${ }^{34,35}$ In this method we used fourth instar larvae of Spodoptera litura of an insect which are responsible for the damage of Indian agricultural crops. Ten larvae were used for each replication and three replications were maintained for each compound. The given compounds were dissolved in acetone. The leaf disc were prepared out of caster leaf and dipped in various test compounds for thirty seconds. The leaf discs were air dried to evaporate the excess acetone. (The leaf discs dipped only in acetone were served as control). The mortality data was recorded after $24 \mathrm{hrs}$, and the treatment mortality were corrected with control mortality. These mortality data was used for calculation of $\mathrm{LC}_{50} / \mathrm{LD}_{50}$ using maximum likelihood program, MLP 3.01. The results of Stomach Toxicity are summarized in Table 6.

\section{Results and Discussion}

Dimethyl (substituted-1,3-benzothiazol-2-yl)dithioimido- 
carbonates (2) have been synthesized by the reaction of substituted-2-aminobenzothiazoles (1) with carbon disulphide, sodium hydroxide and methyl iodide in ice cold solution with DMF as solvent. Further $N$-(1H-benzimidazol-2-yl)-substituted-1,3-benzothiazol-2-amines (3) and substituted- $N$-(4,5dihydro-1H-imidazol-2-yl)-1,3-benzothiazol-2-amines (4) were synthesized by the reaction of compound $\mathbf{2}$ with $o$-phenylene diamine and ethylene diamine respectively. The structures of all the synthesized compounds were established on the basis of spectroscopic and analytical data. The elemental analysis $(\mathrm{C}$, $\mathrm{N}$ and $\mathrm{H}$ ) found for all the condensed products were in close agreement with the calculated values, the infrared (IR) spectrum of compounds 3 and $\mathbf{4}$ display two characteristic bands at $3350-3250$ and $2910-2860 \mathrm{~cm}^{-1}$ due to N-H and $\mathrm{CH}_{2}$ vibrations, respectively.

Antibacterial Activity. The antibacterial activity of all the synthesized compounds were tested in-vitro against pathogenic Escherichia coli, Klebsiella species, Micrococcus luteus and Staphylococcus aureus at $200 \mu \mathrm{g} / \mathrm{mL}$ and $100 \mu \mathrm{g} / \mathrm{mL}$, and the results were compared with some standard drugs like Novobiocin, Kanamycin and Amikacin. In case of $E$. coli Compounds 3A, 3B, 4A and 4B exhibited higher activity at $200 \mu \mathrm{g} / \mathrm{mL}$ while the rest of compounds showed moderate activity. In case of Klebsiella spp. compounds $\mathbf{3 B}$ and $\mathbf{4 B}$ showed higher activity than the rest of compounds. In case of Micrococcus lutius and $S$. aureus compounds $\mathbf{3 A}, \mathbf{4 A}, \mathbf{4 B}$ and $\mathbf{3 B}$ show higher efficacy than rest of compounds. The presence of halogen groups, in $\mathbf{3 A}$, 3B, 4A and 4B play an important role in activity. Sometimes these compounds when present in low concentrations may cause bacteriostatic conditions which slow down the growth of bacteria (Table 2).

Antifeedant Activity. The Antifeedant activity of the newly synthesized compounds was tested by leaf dip method ${ }^{34,35}$ against larvae of Spodoptera litura. The results clearly indicate that the compounds show higher, moderate and less antifeedant activity against the larvae of the insect. Compounds 3A, 3D, 3E and 4A showed higher activity, compounds 3C, 4B and 4D showed moderate activitie. It may be found that these compounds may cause a spasm condition in insects by interacting with the active site of the enzyme responsible for nervous breakdown in insects (Table 3).

Acaricidal Activity. The Acaricidal activity of these compounds was performed by the same method, as in the case of Antifeedant activity, against Tetranychus urticae, a species of mite using acetone as a standard. The results obtained clearly show that compound 3C, 3E, 4C and 4D showed the highest acaricidal activity with respect to other compounds. It is reported that the compounds which are easily soluble in polar solvent have higher activity against microbes and insect, pests or mites. ${ }^{41}$ Rest compounds showed moderate acaricidal activity against the mites (Table 4).

Contact Toxicity. The contact toxicity of these compounds was carried out by topical application method ${ }^{36,37}$ against larvae of Spodoptera litura. The results clearly indicate that the compounds show moderate and lower contact toxicity against the larvae of the insect. Compounds 3B, 3D, 3E, 4A, 4D and 4C showed moderate activity and the rest of the compounds showed lower to moderate activity against the mites (Table 5).
Stomach Toxicity. The stomach toxicity of these compounds was carried out by leaf dip method. ${ }^{34,35}$ In this method we used fourth instar larvae of Spodoptera litura. The results clearly indicate that the compounds show moderate and lower stomach toxicity against the larvae of the insect. Compounds $\mathbf{3 B}, \mathbf{3 E}$, 4A and 4B showed moderate activity and the rest of the compounds showed lower to moderate activity against the mites (Table 6).

\section{Conclusions}

All the newly synthesized compounds were screened for antibacterial activity at a concentration of $200 \mu \mathrm{g} / \mathrm{mL}$ and 100 $\mu \mathrm{g} / \mathrm{mL}$ using DMF as a control. Novobiocin, Kanamycin and Amikacin were used as standard against gram positive and gram negative bacteria. The data in the Table 2 indicates that among the synthesized compounds $\mathbf{3 A}, \mathbf{3 B}, \mathbf{4 A}$ and $\mathbf{4 B}$ posses a broad spectrum activity. However, the activities of the tested compounds are much less than those of standard antibacterial agents used. Antifeedant activity, contact toxicity and stomach toxicity against Spodoptera litura and acaricidal activity against Tetranychus urticae were moderate for test compounds. From the results, it is clear that these compounds would be of better use in drug development to combat bacterial infections and as pesticides in future.

Acknowledgments. The authors are thankful to Head, Department of Chemistry, Govt. College, Ajmer for providing necessary laboratory facilities. We are also grateful to Dr. Ashish Bhatnagar, Head, Department of Microbiology, M. D. S. University, Ajmer for facilities provided to carry out antibacterial studies.

\section{References}

1. Besson, T.; Benetau, V.; Guillard, J.; Leonce, S.; Pfeiffer, B. J. Med. Chem. 1999, 34, 1053.

2. Caleta, I.; Gridisa, M.; Mrovs, S. D.; Cetina, M.; Tralic, K. V.; Pavelic, K. Il Farmaco. 2004, 59, 297.

3. Suvarna, K.; Swain, S, P.; Gandhi, A, M. Indian J. Pharm. Sci. 2007, 69(1), 46.

4. Shastry, C. S.; Joshi, S. D.; Aravind, M. B.; Veerapur, V. P. Ind. J. Het. Chem. 2003, 13, 57.

5. Yilidiz-Oren, I.; Yalcin, I.; Aki-Sener, E.; Ucar, Turk, N. Eur. J. Med. Chem. 2004, 39, 291.

6. Latrofa, A.; Franco, M.; Lopedota, A.; Rosato, A.; Carone, D.; Vitali, C. Il Farmaco. 2005, 60, 291.

7. Kaur, H.; Kumar, S.; Singh, I.; Saxena, K. K.; Kumar, A. Digest J. Nanomaterials and Biostructures 2010, 5(1), 67.

8. Oketani, K.; Nagakura, N.; Harada, K. T. Eur. J. Pharmacol. 2001, 422, 209.

9. Paramashivappa, R.; Phani, K. P.; Rao, P. V. S.; Rao, S. Bioorg. Med. Chem. Lett. 2003, 13, 657.

10. Nagarajan, S, R.; De, C. G. A.; Getman, D. P.; Lu, H. F.; Sikorski, J. A.; Walker, J. L. Bioorg. Med. Chem. Lett. 2003, 11, 4769.

11. Florence, D.; Antonio, A.; Carole, D, G.; Maxime, R.; Erik, D. C.; Pierre, T. Eur. J. Med. Chem. 2004, 39, 685.

12. Yang, B. Q.; Yang, P. H.; Zhu, A. L. Chinese Chemical letters 2003, 14(9), 901.

13. Saggu, J. S.; Sharma, R.; Dureja, H.; Kumar, V. J. Indian Inst. Sci. 2002, 82, 177.

14. Ayhan, K. G.; Kus, C.; Coban, T.; Can, Eke, B. J. Enzyme Inhib. 
Med. Chem. 2004, 19(2), 129.

15. Tewari, A. K.; Mishra, A. Indian J. Chem. 2006, 45(B), 489.

16. Mahajan, S. S.; Nandre, R. G. Indian J. Chem. 2006, 45(B), 1759.

17. Le, H. T.; Lemaire, I. B.; Gilbert, A. K.; Jolicoeur, F.; Yang, L.; Leduc, N. JPET 2004, 309, 146.

18. Rao, A.; Chimirri, A.; Ferro, S.; Monforte. A. M.; Monforte, P.; Zappalà, M. ARKIVOC 2004, 147.

19. Navarrete, V. G.; Cedillo, R.; Hernandez, C. A.; Yepez, L.; Valdez, J.; Morales. R.; Coryes, R.; Catillo, R. Bioorg. Med. Chem. Letter 2001, 11(2), 187.

20. Evers, D. L.; Gloria, K.; Ptak, R. G.; Emmer, B. T.; Shin, D.; Drach, J. C. Antimicrobial agents \& chemotherapy 2004, 48(10), 3918.

21. Kilcigil, G. A.; Altanlar, N. Turk. J. Chem. 2006, 30, 223.

22. Nawrocka, W. Boll Chim. Farm. 1996, 135(1), 18.

23. Rivara, M.; Zuliani, V.; Cocconcelli, G.; Morini, G, V.; Comini, M.; Rivara, S. Bioorg. Med. Chem. Lett. 2006, 14(5), 1413.

24. Shaya, Y. A. R.; Ahmed, M. S.; Elsharief, Saied, M. E. K.; Abdulkhaliq, M. A. A. Heteroatom Chem. 2006, 17(7), 634 \& ChemInform. 2007, 38, 12.

25. Pandey, J.; Tiwari, V. K.; Verma, S. S.; Chaturvedi, V.; Bhatnagar, S.; Sinha, S.; Gaikwad, A. N.; Tripathi, R. P. Eur. J. Med. Chem. 2009, 44, 3350

26. Dahiya, R.; Kumar, A. E. J. Chem. 2008, 5, 1133.
27. Banfi, E.; Scialino, G.; Zampieri, D.; Mamolo, M. G.; Vio, L.; Ferrone, M.; Fermeglia, M.; Paneni, M. S.; Pricl, S. J. Antimicrob. Chemother. 2006, 58, 76.

27. Ulusoy, N.; Gursu, E.; Ozkirimil, S.; Ekinci, A. C.; Dikici, I. Indian J. Chem. 2003, 42(B), 2853.

28. Sharma, D.; Narasimhan, B.; Kumar, P.; Judge, V.; Narang, R.; Clercq, E. D.; Balzarini, J. Eur. J. Med. Chem. 2009, 44(6), 2347.

29. Atia, A. J. K. Molecules 2009, 14, 2431.

30. Ojha, K. G.; Jain, S. K.; Gupta, R. R. Synth. Commun. 1979, 9(6), 457.

31. Gupta, R. R.; Ojha, K. G.; Kumar; M. J. Heterocycl. Chem. 1980, $17,1325$.

32. Cruickshank, R.; Duguid, J. P.; Marmion, B. P.; Swam, H. A. The Practice of Medical Microbiology, 12th Ed.; Churchill Livingstone: London, 1975; p 544.

33. Shelton, A. M.; Robertson, J. L.; Tang, J. D. J. Econ., Entomol. 1993, 86(9), 697.

34. Jinfeng, H.; Pei, L.; Xueyan, S.; Xiwu, G. J. Insect. Sci. 2008, $8(3), 9$.

35. Leonardi, M. G.; Cappelloza, S.; Ianne, P.; Cappelloza, L.; Parenti, P.; Giordana, B. Compedium of Biochemistry and Physiology 1996, $113(\mathrm{~B}), 361$.

36. Ludvik, G. F. J. Econ. Entomol. 1953, 46, 364. 